\title{
Hepatic Transporter Expression in Metabolic Syndrome: Phenotype, Serum Metabolic Hormones, and Transcription Factor Expression ${ }^{\mathbb{S}}$
}

\author{
Ajay C. Donepudi, Qiuqiong Cheng, Zhenqiang James Lu, Nathan J. Cherrington, \\ and Angela L. Slitt
}

\begin{abstract}
Department of Biomedical and Pharmaceutical Sciences, University of Rhode Island, Kingston, Rhode Island (A.C.D., Q.C, A.L.S); Arizona Statistical Consulting Laboratory, The Bio5 Institute (Z.J.L.) and Department of Pharmacology and Toxicology, College of Pharmacy (N.J.C.), University of Arizona, Tucson, Arizona
\end{abstract}

Received September 1, 2015; accepted January 16, 2016

\begin{abstract}
Metabolic syndrome is a multifactorial disease associated with obesity, insulin resistance, diabetes, and the alteration of multiple metabolic hormones. Obesity rates have been rising worldwide, which increases our need to understand how this population will respond to drugs and exposure to other chemicals. The purpose of this study was to determine in lean and obese mice the ontogeny of clinical biomarkers such as serum hormone and blood glucose levels as well as the physiologic markers that correlate with nuclear receptor- and transporter-related pathways. Livers from male and female wild-type (WT) (C57BL/6) and ob/ob mice littermates were collected before, during, and after the onset of obesity. Serum hormone and mRNA levels were analyzed. Physiologic changes and gene expression during maturation and progression to obesity were performed and correlation analysis was performed using canonical
\end{abstract}

correlations. Significant ontogenic changes in both WT and ob/ob mice were observed and these ontogenic changes differ in ob/ob mice with the development of obesity. In males and females, the ontogenic pattern of the expression of genes such as Abcc3, 4, Abcg2, Cyp2b10, and 4a14 started to differ from week 3, and became significant at weeks 4 and 8 in ob/ob mice compared with WT mice. In obese males, serum resistin, glucagon, and glucose levels correlated with the expression of most hepatic ATP-binding cassette (Abc) transporters, whereas in obese females, serum glucagon-like peptide 1 levels were correlated with most hepatic uptake transporters and P450 enzymes. Overall, the correlation between physiologic changes and gene expression indicate that metabolism-related hormones may play a role in regulating the genes involved in drug metabolism and transport.

\section{Introduction}

Obesity is a metabolic disease characterized by an increased body mass index $(\mathrm{BMI} \geq 30$ ). It is a predominant risk factor for metabolic syndrome (MetS), which encompasses increases in body weight, adipose tissue mass, insulin resistance, and serum hormone levels (Grundy, 2004). One of the manifestations of metabolic syndrome is the development of hepatic lipid accumulation (e.g., steatosis) that represents nonalcoholic fatty liver disease (NAFLD) in conjunction with insulin resistance (Wanless and Lentz, 1990). There is a growing concern about absorption, distribution, metabolism, and excretion (ADME) in the obese population, as studies reveal altered ADME in people affected with obesity and metabolic diseases (Brill et al., 2012). Ob/ob mice have a mutation in the

This work was supported by the Rhode Island Foundation, and also supported in part by National Institutes of Health awards to A.S. [1R01ES016042, 5K22ES013782, and 1R15ES025404], and Rhode Island IDeA Network of Biomedical Research Excellence [Award no. P20RR016457-10] from the National Center for Research Resources.

This work was presented in part at the Annual Society of Toxicology meeting, 2010, March 7-11, Salt Lake City, UT.

dx.doi.org/10.1124/dmd.115.066779.

S This article has supplemental material available at dmd.aspetjournals.org. $o b$ gene that encodes for leptin, resulting in a phenotype that has many characteristics common to MetS. Owing to lack of leptin, ob/ob mice exhibit hyperphagia, profound weight gain, hyperglycemia, glucose intolerance, elevated plasma insulin, and severe hepatic steatosis (Lindstrom, 2007). Most of these changes are observed predominantly in ob/ob mice after 3 weeks of age (Dubuc, 1976).

ADME of xenobiotics and several endogenous compounds are regulated by drug-metabolizing enzymes (DME) and drug transporters. Drug transporters are membrane-bound proteins that facilitate both uptake and efflux of xenobiotics, endogenous compounds, and their metabolites in various tissues, including liver. Hepatic transporter expression is an important determinant in maintaining systemic balance of endogenous compounds such as bile acids, hormones, and bilirubin (Lecureux et al., 2009). Multiple conditions can alter drug transporter expression in liver, such as obesity, oxidative stress, inflammation, drug-induced liver injury, and environmental toxicants (Geier et al., 2003; Aleksunes et al., 2008; Cheng et al., 2008). Previous studies document alterations in DME and drug transporter expression in obese and diabetic conditions (Cheng et al., 2008; More and Slitt, 2011).

In liver, transcription factors such as pregnane- $\mathrm{X}$ receptor (Pxr, Nr1i2), constitutive androstane receptor (Car, Nr1i3), farnesoid X receptor (Fxr, Nr1h4), and nuclear factor E2-related factor 2 (Nrf2,

ABBREVIATIONS: Abc, ATP-binding cassette; ADME, absorption distribution metabolism and elimination; Bsep (Abcb11), bile salt export pump; P450, cytochrome P450; Car (Nr1i3), constitutive androstane receptor; DME, drug-metabolizing enzyme; Fxr (Nr1h4), farnesoid X receptor; GLP, glucagon-like peptide; Lxr (Nr1h3), liver X receptor; MetS, metabolic syndrome; NAFLD, nonalcoholic fatty liver disease; Nrf2 (Nfe2I2), nuclear factor E2-related factor 2; Ntcp (Slc10a1), $\mathrm{Na}^{+}$-taurocholate cotransporting polypeptide; Ppar- $\alpha$, peroxisome proliferator-activated receptor alpha; Ppargc1 $\alpha$, peroxisome proliferator-activated receptor gamma coactivator 1-alpha; Pxr (Nr1i2), pregnane-X receptor; WT, wild type. 
$\mathrm{Nfe} 212$ ) regulate the basal and inducible expression of biotransformation enzymes and ATP-binding cassette $(\mathrm{Abc})$ transporters (Klaassen and Slitt, 2005). For example, Pxr and Car upregulate Cyp3a11 and Cyp2b10 expression, whereas Nrf2 upregulates Nqo1 and Gst gene transcription and expression (Aleksunes et al., 2006). With regard to transporters, hepatic Abcc2-4 induction by microsomal enzyme inducers is observed to be Nrf2-dependent (Maher et al., 2005). Prototypical Pxr activators upregulate hepatic Abcc2, Abcc3, $\mathrm{Na}^{+}-$ taurocholate cotransporting polypeptide (Ntcp), and solute-carrier organic anion transporter (Slco)1a4 expression (Cheng et al., 2005, 2007; Maher et al., 2005), whereas Car activators upregulate Abcc2-6 mRNA expression in liver (Cheng et al., 2005; Maher et al., 2005). An increase in mRNA expression of these transcription factors was observed in livers of 9-week-old ob/ob mice compared with WT mice (Xu et al., 2012), implicating coordinate regulation of drug-transporter and transcription-factor expression in steatosis. However, the correlation or coordinated expression of transcription factors and transporter expression during development of fatty liver disease has not been well described or documented.

Obesity alters levels of several metabolic hormones, such as resistin, glucagon, insulin, and incretins (Starke et al., 1984; Azuma et al., 2003; Reinehr et al., 2007), which may influence hepatic gene expression in obesity. Along with hormonal changes, diabetes and obesity causes insulin resistance accompanied by hyperglycemia, which are known to regulate expression of several hepatic genes (Kahn et al., 2006). Although increased incretin levels, such as glucagon-like peptide-1 (GLP-1), are observed in obese people, GLP-1 activity associated with insulin secretion is decreased compared with lean individuals (Laferrere et al., 2007). Several therapies that target these hormones have been identified for treatment of obesity and other metabolic diseases (Schmitz et al., 2004). Serum levels of such hormones as resistin, leptin, and insulin were correlated to metabolic disease such as obesity (Pantsulaia et al., 2007), indicating obesity can alter levels of hormones involved in regulation of metabolism, or vice-versa. As more endocrine hormone-targeting drugs are being approved and developed to manage aspects of metabolic disease, there is a need to understand the influence of these hormones on processes that dictate hepatic gene expression involved in drug metabolism and disposition in both preclinical species and humans.

Leptin-deficient obese (ob/ob) mice are commonly used to model MetS and fatty liver disease (Lindstrom, 2007). This study aimed to correlate typical clinical endpoints such as metabolic hormones and physiologic factors with hepatic transcription factors, prototypical DME, and transporter-mRNA expression to better predict drug disposition. The findings of this study provide potential insight into possible measures and serum biomarkers that could be associated with ADME changes in obesity.

\section{Materials and Methods}

Animals and Husbandry. Adult heterozygous Lep ${ }^{\mathrm{ob} /+}\left(\mathrm{OB}, \mathrm{B} 6 . \mathrm{V}-\mathrm{Lep}^{\mathrm{ob}} / \mathrm{J}\right.$, stock no. 0000632) breeders were purchased from Jackson Laboratories (Bar Harbor, ME). Heterozygous breeders were mated and offspring were genotyped for sex and mutation of the leptin gene. The resulting wild-type or C57BL/6 mice (WT) used in this study were considered the WT controls and leptin-deficient homozygous mice were considered obese (ob/ob) mice. In ob/ob mice pronounced changes in physiologic factors have been observed from 3 weeks of age (Dubuc, 1976), so to identify correlation between physiologic factors and hepatic gene expression during development of obesity we chose mice of different ages, such as week 1 (pre-), week 3 , week 4 (post-), and week 8 (adult) mice. Tissues from male and female WT and ob/ob were collected at 1,3, 4, and 8 weeks $(n=$ 4-5 per group). Blood was collected and serum was obtained after centrifugation at $5000 \mathrm{rpm}$ for 5 minutes at $4^{\circ} \mathrm{C}$. Livers were collected, snap frozen in liquid nitrogen, and stored at $-80^{\circ} \mathrm{C}$ for future analysis. All animal experiments were approved by University of Rhode Island Institutional Animal Care and Use Committee.
RNA Extraction. Total RNA from livers was isolated by phenol-chloroform extraction method using RNA-Bee reagent (Tel-Test, Inc., Friendswood, TX), according to the manufacturer's protocol. RNA concentration was quantified by absorbance at $260 \mathrm{~nm}$ using a Nanodrop ND1000 (Thermo Fisher Scientific, Waltham, MA) and the samples were diluted to $1 \mu \mathrm{g} / \mu \mathrm{l}$. Formaldehyde-agarose gel electrophoresis followed by UV illumination was used to visualize $28 \mathrm{~S}$ and $18 \mathrm{~S}$ ribosomal RNA and confirm integrity.

QuantiGene Multiplex Suspension Array. Mouse liver mRNA expression was determined using a QuantiGene Plex 2.0 assay (Panomics/Affymetrix eBioscience, San Diego, CA) with a Bio-Plex System array reader with Luminex 100 xMAP technology, and data were acquired using Bio-Plex Software Data Manager (Bio-Rad, Hercules, CA). Assays were performed according to the manufacturer's protocol. The optimal RNA input was determined prior to running the assay. Briefly, $500 \mathrm{ng}$ of total RNA was incubated overnight at $53^{\circ} \mathrm{C}$ with xMAP beads containing oligonucleotide capture-probes, label extenders, and blockers. On the next day, beads and bound target RNA were washed and subsequently incubated with amplifier at $46^{\circ} \mathrm{C}$ for 1 hour. Samples were then washed and incubated with the label (biotin) at $46^{\circ} \mathrm{C}$ for 1 hour. Samples were washed with streptavidin-conjugated R-phycoerythrin, which binds biotinylated probes, and incubated at room temperature for 30 minutes. Streptavidin-conjugated R-phycoerythrin fluorescence was then detected for each analyte within each sample.

Branched DNA Amplification Assay. Relative expression of bile saltexport pump (Abcb11, Bsep) and $\mathrm{Na}^{+}$-taurocholate cotransporting polypeptide (Slc10a1, Ntcp) mRNA was quantified using bDNA assay and previously described probe sets (Cheng et al., 2007). All reagents for analysis, including lysis buffer, amplifier/label probe diluent, and substrate solution, were supplied in the QuantiGene 1.0 assay kit (Panomics/Affymetrix eBioscience). Briefly, the probe-set stocks containing capture extenders, label extenders, and blockers were diluted 1:100 in lysis buffer before use. On day 1, total RNA samples $(10 \mu \mathrm{g})$ were added to wells containing $50 \mu \mathrm{l}$ of capture hybridization buffer and $50 \mu \mathrm{l}$ of diluted probe set. The RNA was allowed to hybridize overnight with the probe set at $53^{\circ} \mathrm{C}$. On day 2 , subsequent hybridization steps were followed as detailed in the manufacturer's protocol, and fluorescence was measured with a GloRunner Microplate Luminometer interfaced with GloRunner DXL Software (Turner BioSystems, Sunnyvale, CA). The fluorescence for each well was recorded as relative light units (RLU) (Donepudi et al., 2012).

Serum Metabolism-Related Hormone Levels. Serum metabolism-related hormones were quantified using a Millipore 10-plex kit (MMHMAG-44K; EMD Millipore/Merck, Billerica, MA ) on a Bioplex multiple array system. A custom Millipore-plex kit containing different targets such as insulin, glucagon, resistin, GLP-1, amylin and leptin was used and analyzed according to manufacturer's protocol. Fluorescence was detected on a Bioplex multiple array reader system (BioRad). Data were collected by Bioplex Software Manager 5.0 and plotted as average concentration $(\mu \mathrm{g} / \mathrm{ml})$. Serum glucose levels were analyzed using a Glucose Colorimetric Assay Kit (Cayman Chemical, Ann Arbor, MI).

Correlation Analysis. Correlations between the mRNA levels of genes related to drug metabolism and transcription factors were performed using either Statistica 9.1 software (StatSoft, Inc., Tulsa, OK) or canonical correlation analysis. Briefly, for canonical correlation analysis data generated was logtransformed and distributed in three blocks such that block 1 contained gene expression of drug transporters and phase I enzymes, block 2 contained transcription factor expression, and block 3 contained physiologic dataserum hormone and glucose levels and body and liver weights. Cross-block pairwise bivariate correlations were performed between each block, and heat maps were generated. Hierarchical clustering was performed using the same data with Pearson correlation. Data presented as heat maps or with $r$-value $P \leq 0.05$ were considered a statistically significant correlation.

Statistical Analysis. The statistical significance between groups was determined by factorial analysis of variance (ANOVA) followed by a Duncan multiple-range post-hoc test, using Statistica 9.1 software (StatSoft, Inc.). Data are presented as mean \pm S.E., with $P \leq 0.05$ considered statistically significant.

\section{Results}

Ontogeny of Serum Hormones in WT and Ob/Ob Mice. At week 1 of age, body weight was similar between all groups, and at weeks 4 and 8 , ob/ob mice body weight had increased by at least 1.2- and 1.7-fold, respectively, compared with WT mice (Supplemental Data, Fig. 1A). 
Serum glucose levels increased from week 1 to 8 in both males and females (Supplemental Data, Fig. 1B). In ob/ob mice, serum glucose levels increased with age from week 3 in females and week 4 in males compared with week-1 ob/ob mice. Figure 1 illustrates serum metabolic hormone changes observed in both male and female WT and ob/ob mice from weeks 1, 3, 4, and 8. Interestingly, in both male and female WT mice such serum hormone levels as glucagon, resistin, and GLP-1 changed with age. However, in both male and female WT mice serum insulin and amylin levels were similar at all ages. In WT mice, serum glucagon, resistin, and GLP-1 levels were decreased by $\sim 78 \%$ from week 3 compared with week 1 . These age-dependent changes in serum glucagon and GLP-1 levels were not observed in either male and female ob/ob mice.

In both male and female mice, serum hormone levels were similar between WT and ob/ob mice at 1 week of age. However, in both male and female ob/ob mice, serum insulin and amylin increased with age after week 4 by 4 -fold compared with week 1 , whereas glucagon, resistin, and GLP-1 levels decreased by 57,31 , and $40 \%$ respectively. In both males and females, all serum hormone levels were significantly increased in ob/ob compared with 8-week-old WT mice.

Hepatic Drug Transporters and Prototypical MetabolizingEnzyme Expression in Livers of WT and $\mathrm{Ob} / \mathrm{Ob}$ Mice. Figure 2A illustrates hepatic efflux drug transporter expression observed in both male and female WT and ob/ob mice. In WT and ob/ob mice, ontogenic changes were observed in expression of Abc transporters such as Abcc1, 3, 4, 5, and Bsep levels in both males and females. At week 1, Abc transporter expression was similar between male and female WT and ob/ob mice. In male ob/ob mice at week $8, \mathrm{Abcc} 3,4$, and Abcg2 mRNA expression were higher than WT mice by 1.8-, 7-, and 2.3-fold, respectively. A similar increase was observed in female ob/ob mice compared with WT mice in Abcc3, 4, and Abcg2 mRNA expression. In contrast to males, during weeks 4 and 8 expression of Bsep mRNA in female ob/ob mice decreased by $49 \%$.

Figure 2B illustrates hepatic uptake drug transporter expression in both male and female WT and ob/ob mice. In WT male and female mice, Slcola1 expression was 219- and 95-fold higher in week 8 compared to week 1 counterparts, respectively. Interestingly, this increase in Slcola1 mRNA levels at week 8 was not observed in both male and female ob/ob mice. In week 8 male and female ob/ob mice, Slcola1 expression had decreased to about $3 \%$ of the WT counterparts. In male and female WT and ob/ob mice, no ontogenic changes were observed in expression of hepatic uptake transporters such as Slco1a4, 1b2, and Slc10a1, although an increased expression of Slcola4 was observed only at 3 and 4 weeks compared with respective week 1 counterparts.

Figure 2C illustrates hepatic DME expression in WT and ob/ob mice. In both male and female mice, Cyp3a11 mRNA expression increased with age, with no significant changes between ob/ob and WT counterparts at the same ages. In male and female ob/ob mice, Cyp2b10 and $4 \mathrm{a} 14$ mRNA expression increased from week 1 to 8 , whereas no significant ontogenic changes were observed with age in WT mice. In males, Cyp2b10 and 4a14 expression in ob/ob mice were 4.3- and 2.2-fold higher than their WT counterparts, respectively. In females, Cyp4a14 mRNA expression in female ob/ob mice was 2- to 3-fold higher than WT counterparts at weeks 4 and 8 . In female ob/ob mice significant increase in Cyp3a11 and Cyp2b10 gene expression by 1.3- and 1.8-fold was observed only at week 4 compared with respective WT counterparts. These changes in Cyp3a11 and Cyp2b10 expression in female ob/ob mice were not observed in week 8 mice.

Hepatic Transcription Factor Expression in WT and ob/ob Mice. In male and female, WT and ob/ob mice, there were no significant ontogenic changes in transcription factor mRNA expression with the exception of Pxr. Figure 3 illustrates the hepatic transcription factor mRNA expression levels in livers of male and female WT and ob/ob mice. In WT female mice, Pxr mRNA expression decreased with age by $51 \%$ compared with week 1 , whereas ontogenic changes in $\mathrm{Pxr}$ expression were not observed in female ob/ob mice. In male ob/ob mice, Nrf 2 mRNA expression was 2 -fold higher at week 8 compared with week 1 counterparts. Male ob/ob mice have 2- to 3-fold higher mRNA levels of liver $\mathrm{X}$ receptor (Lxr), Fxr, and Nrf2 compared with their WT counterparts only at week 8 . These changes in male ob/ob

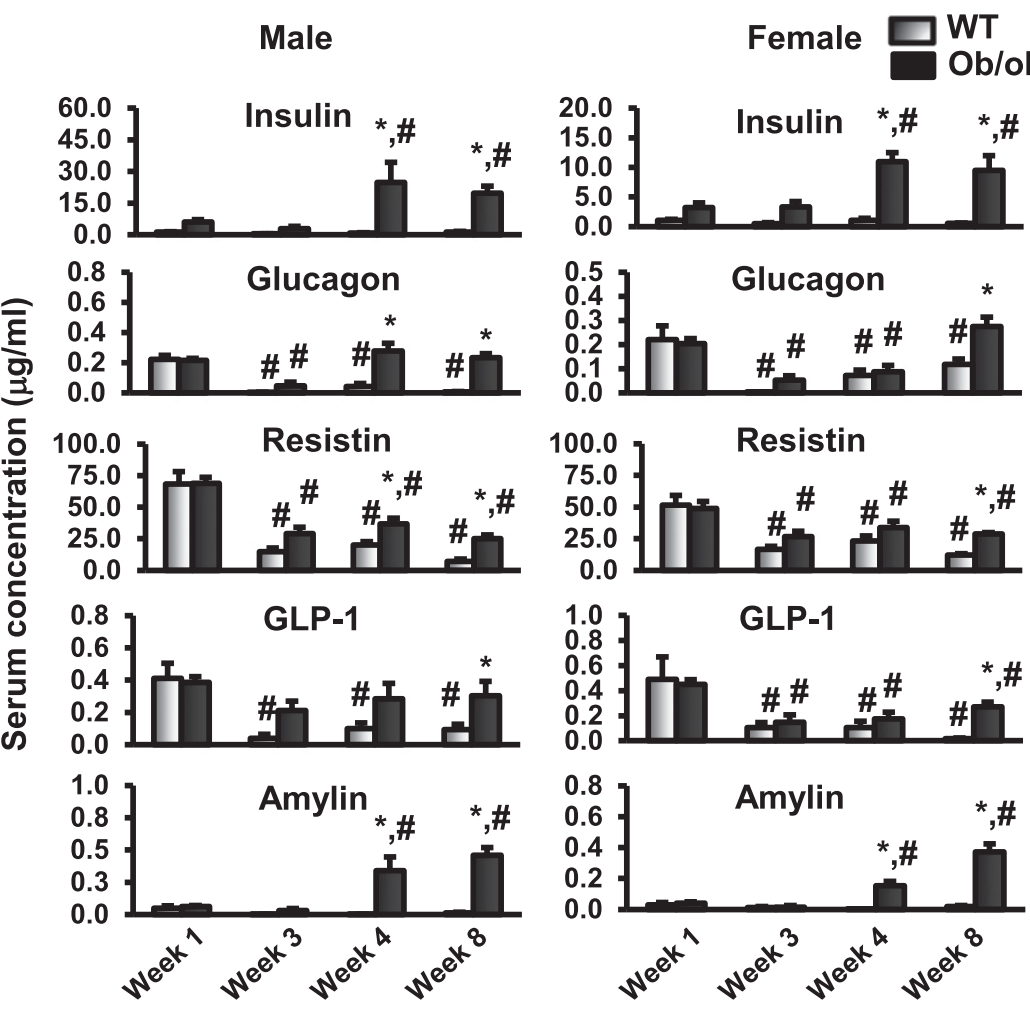

Fig. 1. Effect of age and leptin deficiency on phenotypic changes in WT and ob/ob mice. Metabolism-related hormone levels of WT and ob/ob mice at week-1, 3, 4 and 8. Metabolism-related hormone levels in WT and ob/ob mice were quantified in serum collected from WT and ob/ob mice at $1,3,4$, and 8 weeks of age ( $n=4-5$ for each group/ sex). Hormones were quantified using a Luminex-based assay. Data are presented as average concentration $(\mu \mathrm{g} / \mathrm{ml}) \pm$ S.E.M. $(n=4-5$ mice per group). Asterisks $(*)$ represent a statistical difference between wild type and ob/ob of same age group and hash mark (\#) represent a statistical difference with respect to week-1 mice $(P \leq 0.05)$ 
A

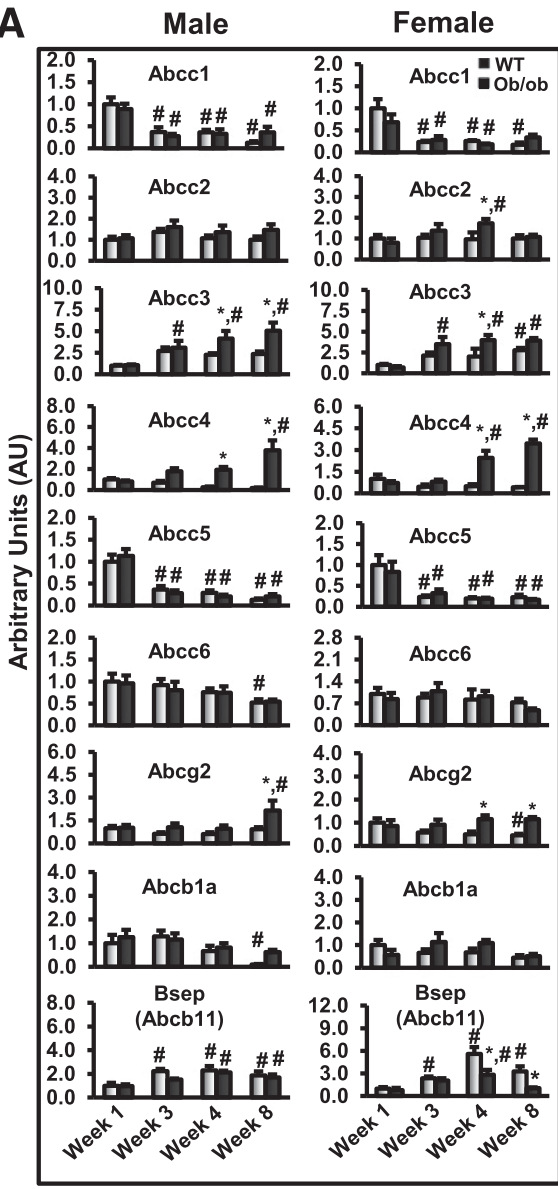

B
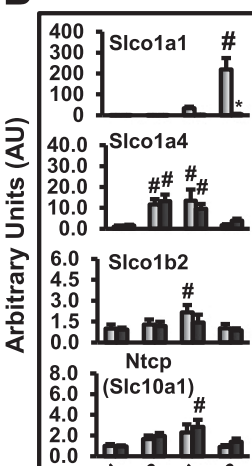

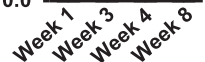

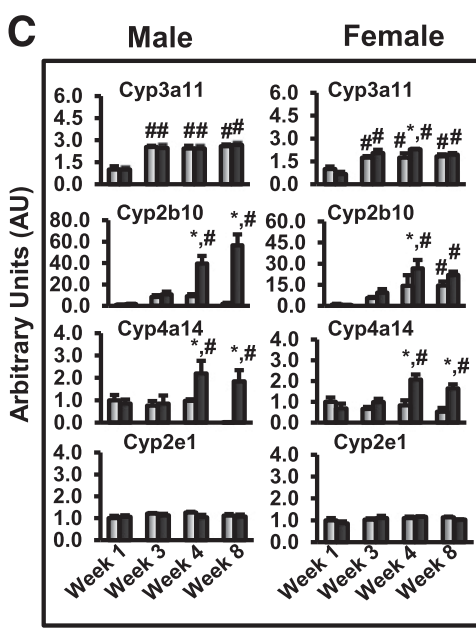

Female

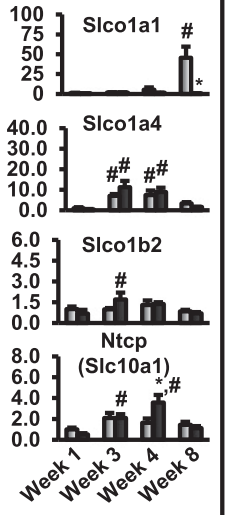

Fig. 2. Age and leptin deficiency alters hepatic drug transporter and biotransformation enzyme expression in WT and ob/ob mice. Total RNA was isolated from livers of WT and ob/ob mice. (A) Hepatic efflux transporter expression. ATP-binding cassette (Abc) transporter mRNA levels were quantified by the QuantiGene Plex (QGP) 2.0 assay. Data are presented as mean arbitrary units (AU) \pm S.E.M. ( $n=4-5$ mice per group/sex). (B) Hepatic uptake transporter expression. Organic anion transport polypeptides (Oatps, Slcos) and $\mathrm{Na}^{+}$-taurocholate cotransporting polypeptide (Slc10a1, Ntcp) mRNA levels were quantified by the QGP 2.0 assay. Data are presented as mean arbitrary units (AU) \pm S.E.M. ( $n=4-5$ mice per group/sex). (C) Hepatic biotransformation enzyme expression. mRNA expression of Cyp3a11, 2e1, 2b10, and 4a14 was quantified with QGP 2.0 assay. Data were presented as fold change of gene expression compared with WT week-1 gene expression of respective gene and indicated as mean arbitrary units $(\mathrm{AU}) \pm$ S.E.M. $(n=4-5$ mice per group/sex $)$. Asterisks $(*)$ represent a statistical difference between wild type and ob/ob of same age group and hash mark (\#) represent a statistical difference with respect to week-1 mice $(P \leq 0.05)$.

mice at week 8 were not observed in female ob/ob mice. In female ob/ob mice at weeks 3 and 4 significant changes in Lxr, Car, and Fxr were observed compared with WT counterparts, but these changes were not observed at the later time point.

Correlation Analysis between Metabolic Hormones and Phenotypic Changes. The role of sex and leptin in ontogenic changes in hepatic gene expression pattern is depicted pictorially using heat maps (Fig. 4). Gene expression values from male and female, and WT and ob/ob mice were log-transformed to generate heat maps for each group. These heat maps strongly indicate that leptin and sex regulate hepatic genes involved in metabolism and disposition. Differences in ontogenic pattern of hepatic gene expression between ob/ob mice and WT mice illustrate the influence of leptin on hepatic gene expression patterns. In WT males, hepatic transcription factors and efflux transporters, with the exception of Abcc2, 3, and Bsep, showed high expression (red color) at week 1 with gradual decrease in expression with age (low expression indicated with green color at week 8), whereas the same expression patterns were not observed in male ob/ob mice. In females also only WT mice had high expression (red color) of efflux transporters, with the exception of Abcc2, 3 , and Bsep, at week 1 and low expression (green color) at week 8. Only a few hepatic transcription factors such as peroxisome proliferatoractivated receptor gamma coactivator 1-alpha $(\operatorname{Ppargc} 1 \alpha)$, and $\mathrm{Pxr}$ showed similar patterns in both male and female WT mice. Table 1 illustrates correlations between physiologic factors, such as metabolic hormone levels, serum glucose, and body and liver weights, that were performed using canonical correlations. Canonical correlations were performed to determine how different metabolic hormones are correlated with each other during the development of obesity (Table 1). In males, only serum resistin and insulin levels correlated with all clinical markers, whereas in female mice, serum resistin and amylin correlated with most other physiologic factors during development of obesity. In both males and females, the correlations between physiologic factors suggest that changes in serum resistin levels may be associated with development of obesity, irrespective of sex.

Correlation Analysis between Gene Expression Changes and Phenotypic Changes. Furthermore, canonical correlations were performed between phenotypic changes observed during development of obesity and gene expression. These correlations were performed to uncover relationships between gene expression during development of obesity and common measurements used to classify MetS. Figure 5 and (Supplemental Table 2 illustrate correlation of physiologic measurements, such as serum hormone levels and body and liver weights, with drug transporter and DME expression. In both males and females, serum amylin levels were correlated with Abcc4, Abcg2, Cyp2b10, and $4 \mathrm{a} 14$ expression during development of obesity. With the exception of amylin, correlation between physiologic factors and drug transporters expression differ between males and females during development of obesity. In male mice, serum resistin levels were correlated with Abcc1, 4, 5, Abcb1a, Bsep, Slco1a1, Cyp3a11, and 4a14 expression, whereas in females they were correlated to Abcc1, Bsep, Slco1a1, 1a4, Ntcp, Cyp3a11, 2b20, and 2e1. Furthermore, serum insulin levels were correlated to Abcc4, Abcg2, Cyp2b10, and 4a14 expression in males and Abcc4, Abcg2, and Slco1a1 expression in females. Serum glucagon concentrations were correlated with Abcc1, 4, Abcg2, Abcb1a, Bsep, Slco1a1, Cyp3a11, and Cyp4a14 expression in males, and in females they are correlated to Abcc6, Bsep, Slco1a4, 1b2, and Ntcp expression. 


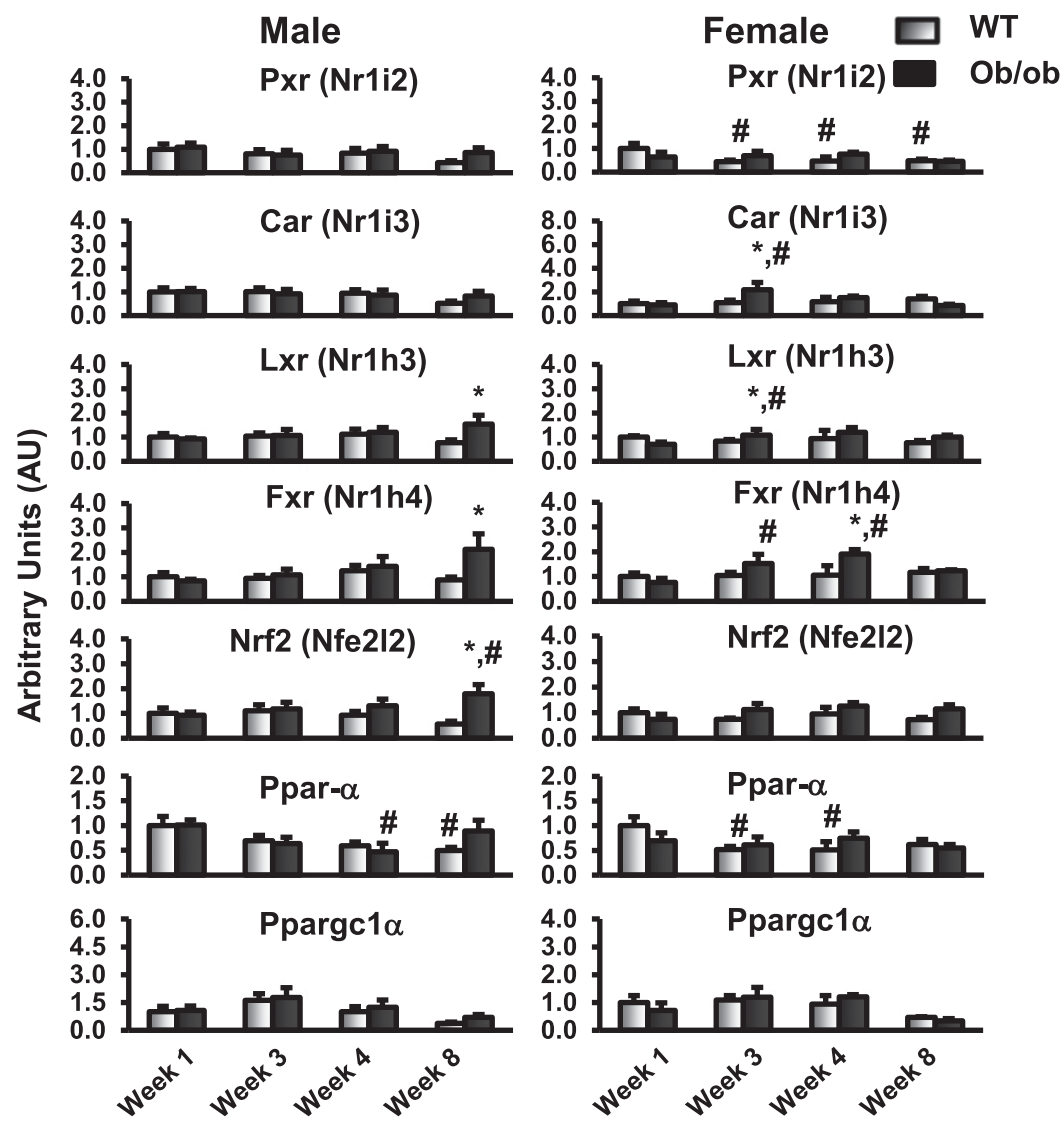

Fig. 3. Effect of age and leptin deficiency on hepatic transcription factor expression in WT and ob/ob mice. Total RNA was isolated from wild-type and ob/ob mice livers, and mRNA expression of Pxr, Car, Lxr, Fxr, Nrf2, Ppar- $\alpha$, and Ppargc1 $\alpha$ was analyzed with QGP 2.0 assay. Data were presented as fold change of gene expression compared with WT week-1 gene expression of respective genes and indicated as mean arbitrary units (AU) \pm S.E.M. $(n=4-5$ mice per group/sex). Asterisks (*) represent a statistical difference between wild-type and ob/ob of same age group and hash mark (\#) represent a statistical difference with respect to week-1 mice $(P \leq 0.05)$

In males, serum GLP-1 concentrations were correlated with only Bsep, Slco1a4, Cyp3a11, and 2e1 expression, whereas in females they were correlated with Abcc2, 3, Bsep, Slco1a1, 1a4, 1b2, Cyp3a11, 2b10, and $2 \mathrm{e} 1$ expression. In males, serum glucose levels were correlated to Abcc1, 3, 5, 6, Abcb1a, Bsep, Cyp3a11, and 2b20 expression, whereas they were correlated to Abcc3-5, Cyp3a11, and 2b10 in females. Both body and liver weights correlated to Abcc1, 3, 5, 6, Abcb1a, Bsep, Slco1a1, Cyp3a11, and 2b10 expression in males, whereas in females they correlated with Abcc1, 3-6, Cyp3a11, and 2b10 expression.

Together these analyses indicate that correlation between physiologic factors and hepatic gene expression differs between males and females during development of obesity. In males, during development of obesity serum resistin, glucagon, and glucose levels correlated with most of hepatic Abc transporter expression compared with serum amylin, insulin, and GLP-1 levels. In females, serum glucose showed a higher correlation with hepatic Abc transporter expression than serum metabolism-related hormones. In females, during development of obesity, hepatic uptake transporter mRNA expression correlated with serum levels of resistin, glucagon, and GLP-1 (Fig. 5 and (Supplemental Table 2). Apart from serum metabolism-related hormones, serum glucose levels and body and liver weight stood as a markers for change in gene expression and physiologic factors during development of obesity (Table 1, Fig. 5, (Supplemental Fig. 2, and (Supplemental Table 2).

Figure 6 illustrates the correlation between transcription factors with drug transporters and phase-I enzyme expression in male and female mice. Correlation between transcription factors, drug transporters, and DME expression was performed using canonical correlations, and the data generated was presented in the form of heat maps. In males, during development of obesity and MetS, expression patterns of all Abc transporters with the exception of Bsep were correlated with all transcription factors analyzed, with minor exceptions. In male mice,
Abcc3 expression did not strongly correlate with Pxr, Car, Ppar- $\alpha$, and Ppargc1 $\alpha$ expression, whereas Abcc5 was not correlated with Fxr. Correlation between Abc transporters and transcription factors showed sex-dependent changes during development of obesity. Interestingly in female mice only, Abcc2, 6, and Abcb1a expression correlated with all transcription factors analyzed, whereas $\mathrm{Abcg} 2$ expression was correlated with all transcription factors with the exception of Car during development of obesity. In female mice, Abcc3 expression correlated to Car, Lxr, Fxr, and Nrf2 expression, whereas Abcc4 expression was correlated to Pxr, Lxr, Fxr, and Nrf2 expression. In both male and female mice, uptake transporter Slco1b2 expression correlated to all transcription factor expressions, whereas Slco1a4 expression was correlated with Car, Lxr, Fxr, Nrf2, and Ppargc1 $\alpha$ expression. Expression of DMEs such as Cyp2e1 and $4 \mathrm{a} 14$ was correlated with all transcription factor expressions in both male and female mice. In male mice, Cyp2b10 expression was correlated to Lxr, Fxr, and Nrf2 expression, whereas in females Cyp3a11 and 2 b10 were correlated with Car, Lxr, Fxr, and Nrf2 expression.

\section{Discussion}

Obesity and ensuing MetS is a major health concern in the United States (Grundy, 2004). Ob/ob mice are often used to model MetS and NAFLD because they have elevated multiple serum biomarkers, hepatic steatosis, and hepatic gene-expression changes that are similar to humans with uncontrolled MetS (Lindstrom, 2007). Drug transporter and DME expression were altered during NAFLD and could have resulted in altered drug elimination (Lickteig et al., 2007; Cheng et al., 2008; Barshop et al., 2011; Hardwick et al., 2012). Moreover, changes in hepatic uptake and efflux transporter expression in ob/ob mice are similar to those observed in livers of diet-induced obese mice or humans with steatosis (Cheng et al., 2008; More and Slitt, 2011). 


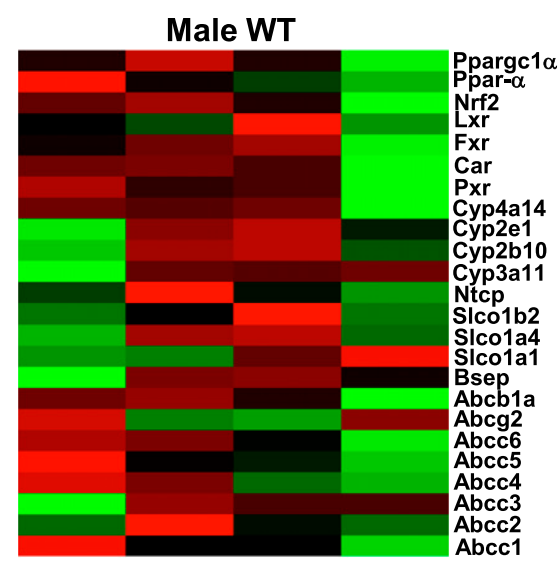

Female WT
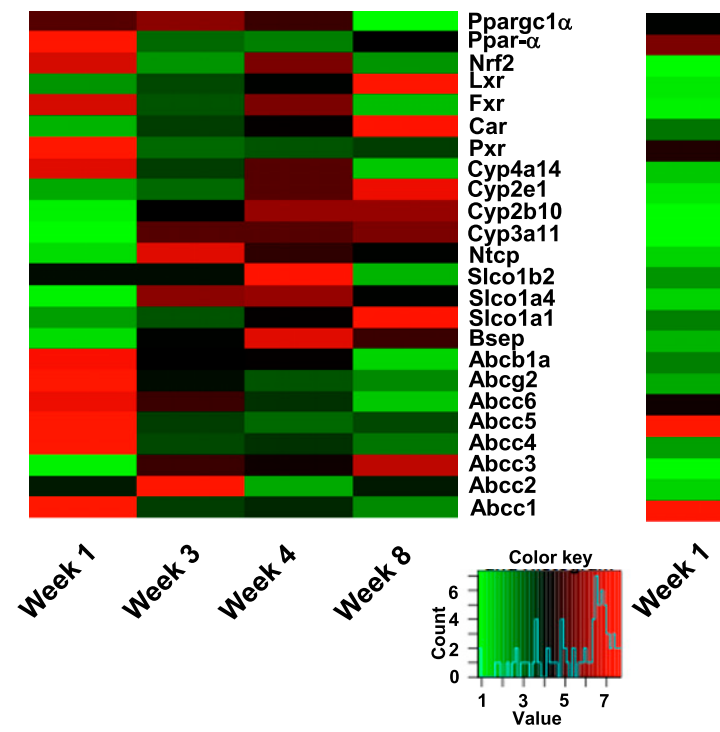

Male ob/ob

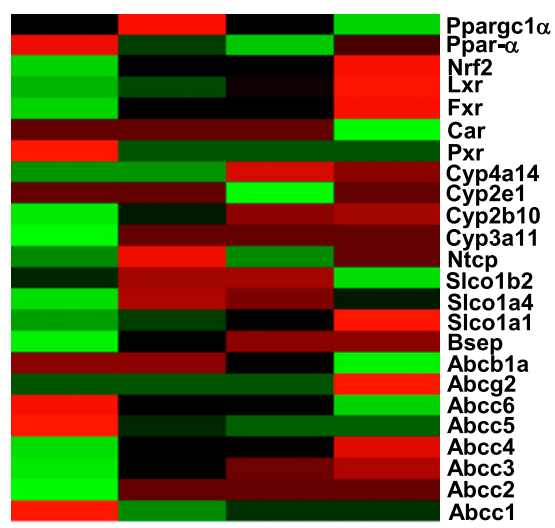

Female ob/ob

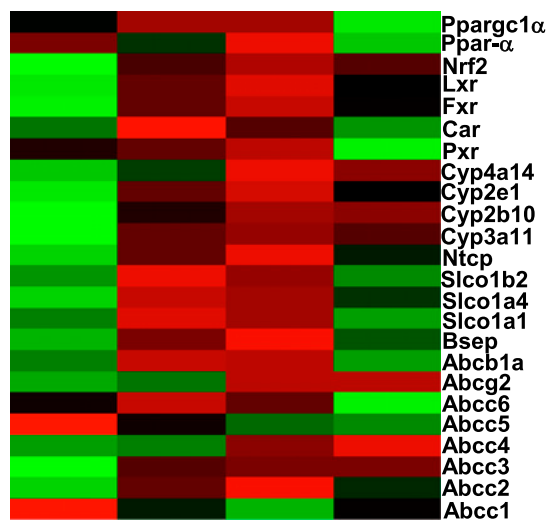

Fig. 4. Heat maps for gene expression analysis performed in both male and female WT and ob/ob mice. Gene expression values are log-transformed and heat maps were generated using $\mathrm{R}$ language.

Abc transporters enable compounds to pass from hepatocytes into bile or blood (Faber et al., 2003). In both WT and ob/ob mice, Abcc1 and 5 were expressed highly at week 1 and decreased with age. This

TABLE 1

The correlation matrices for variables in phenotypical factors during development of obesity in male and female mice

Physiologic factors such as serum metabolism-related hormones, body and liver weight values of WT and ob/ob mice were log transformed and correlation between each other was obtained using canonical correlations. Data presented as $r$ values. *Significant correlation $(\mathrm{P} \leq 0.05)$.

\begin{tabular}{llllllll}
\hline & Amylin & Resistin & Insulin & Glucagon & GLP-1 & Glucose & Body weight \\
\hline Male mice & & & & & & & \\
Resistin & 0.46 & & & & & & \\
Insulin & $0.86^{*}$ & $0.39^{*}$ & & & & & \\
Glucagon & $0.88^{*}$ & $0.75^{*}$ & $0.77^{*}$ & & & & \\
GLP-1 & $0.59^{*}$ & $0.55^{*}$ & $0.57^{*}$ & $0.72^{*}$ & & & \\
Glucose & 0.33 & $-0.45^{*}$ & $0.4^{*}$ & 0.02 & -0.05 & & \\
Body weight & 0.23 & $-0.53^{*}$ & $0.35^{*}$ & -0.11 & -0.13 & $0.94^{*}$ & \\
Liver weight & 0.23 & $-0.52^{*}$ & $0.36^{*}$ & -0.12 & -0.15 & $0.93^{*}$ & $0.99^{*}$ \\
Female mice & & & & & & & \\
Resistin & 0.35 & & & & & & \\
Insulin & $0.62^{*}$ & $0.57^{*}$ & & & & & \\
Glucagon & $0.46^{*}$ & $0.57^{*}$ & $0.52^{*}$ & & & & \\
GLP-1 & $0.37 *$ & $0.75^{*}$ & $0.52^{*}$ & $0.53^{*}$ & & & \\
Glucose & $0.42^{*}$ & $-0.37^{*}$ & 0.25 & 0.13 & -0.28 & & \\
Body weight & $0.47^{*}$ & $-0.38^{*}$ & 0.33 & 0.15 & -0.21 & $0.91^{*}$ & \\
Liver weight & $0.46^{*}$ & -0.34 & $0.38^{*}$ & 0.12 & -0.14 & $0.88^{*}$ & $0.99^{*}$ \\
\hline
\end{tabular}

pattern in expression of Abcc1-high expression at initial stages and decrease at later age-is also observed in liver regeneration after $90 \%$ hepatectomy, indicating that Abcc1 has a lesser role in adult liver (Kimura et al., 2012). Abcc3 and 4 are basolateral efflux transporters with relatively low expression in liver; they are induced by microsomal inducers during several disease conditions (Slitt et al., 2003; Mennone et al., 2006; More and Slitt, 2011). In both obese males and females Abcc 3 and 4 mRNA levels increased with age, and also compared with their WT littermates, whereas no ontogenic changes were observed in Abcc3 and 4 expression with age in WT mice. Apical efflux transporters such as Abcc2, Abcg2, Abcb1a, and Bsep play a major role in excretion of xenobiotics and endogenous substances from liver to bile. In both male and female mice, Abcc2 and Abcbla mRNA levels are unaltered with age and obesity. Previous studies indicated no change in mRNA levels or an increase in protein levels of Abcc2 in ob/ob mice, which is consistent with our results (Cheng et al., 2008). Abcc2 expression during obesity is species-specific; in obese Zucker rats Abcc 2 expression decreases, whereas it increases in ob/ob mice, but in humans there is no change with obesity (Pizarro et al., 2004; Cheng et al., 2008; More and Slitt, 2011). In male ob/ob mice, Bsep expression was similar to respective WT littermates, whereas in females Bsep expression decreased with progression of obesity. The sex-specific difference in Bsep expression was may be attributable to a sex-specific difference in growth hormone responsiveness in obesity (Cocchi et al., 1993), which is known to regulate Bsep expression (Cheng et al., 2007). 
A
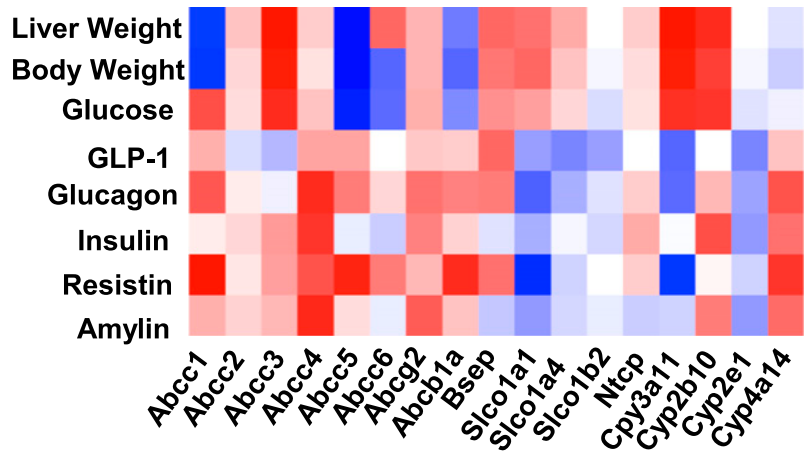

Color key

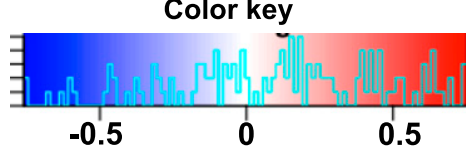

B

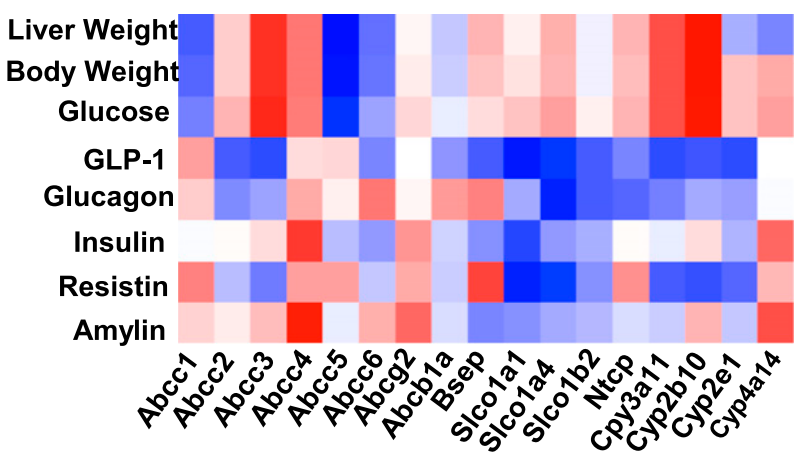

Fig. 5. Correlation between physiologic factors with hepatic drug transporters and biotransformation enzyme expression during development of obesity in (A) male and (B) female mice. Hepatic gene expression from week-1, $-3,-4$, and -8 , WT, and $\mathrm{ob} / \mathrm{ob}$ mice are log-transformed. A log-transformed dataset of both WT and ob/ob was divided into two blocks. Block 1 contains hepatic drug transporters and biotransformation enzyme expression and block 2 contains physiologic factors. Correlations between blocks 1 and 2 were performed using canonical correlations. Data displayed as heat maps, with highest positive correlations considered as red and highest negative correlation are considered as blue.

Previous studies showed that Abc transporter expression is regulated by several transcription factors (Maher et al., 2005). Interestingly, significant correlations were observed between hepatic Abc transporters and transcription factor expression during development of obesity. These correlations during development of obesity between transcription factors and hepatic efflux transporters showed sexdependent changes, and are much more pronounced in week- 8 mice (Supplemental Table 1). In both male and females, hepatic uptake transporter Slcola1 mRNA levels increased with age only in WT mice. In both male and female ob/ob mice Slcola1 mRNA levels decreased significantly at week 8. Previous studies showed that Slco1a1 mRNA expression is androgen-dependent and negatively regulated by microsomal enzyme inducers that activate transcription factors Pxr, Car, Ppar- $\alpha$, and Nrf2 (Lu et al., 1996; Cheng et al., 2005). Ob/ob mice are known to have decreased androgen levels (Swerdloff et al., 1976) and increased transcription factor expression (Xu et al., 2012). Obesity did not affect the ontogeny of Slco1a4, 1b2, and Ntcp in both males and females.

The mRNA expression of phase-I biotransformation enzymes, such as Cyp3a11 and 2e1, was similar between WT and ob/ob mice in this study. Cyp2e1 expression during obesity and diabetes appears to be species-specific. In humans, Cyp2e1 in liver is increased during obesity and diabetes, whereas in mice it appears to be unchanged or decreased
A

Color key
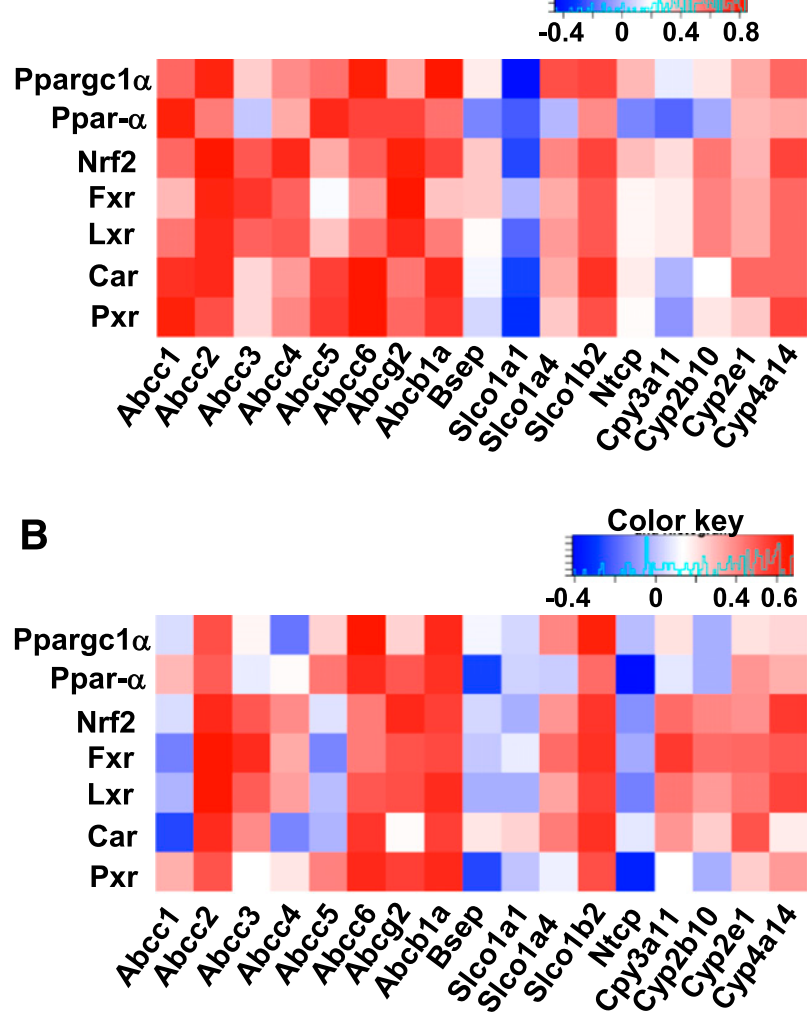

Fig. 6. Correlation between hepatic transcription factors with hepatic drug transporters and biotransformation enzyme expression during development of obesity in (A) male and (B) female mice. Hepatic gene expression, physiologic factors such as body and liver weights, and serum metabolism-related factors such as insulin, glucagon, resistin, and amylin levels, from week-1, $-3,-4$, and -8 , WT, and $\mathrm{ob} / \mathrm{ob}$ mice are log-transformed. The log-transformed dataset of both WT and ob/ob was divided into two blocks, such that one block contained hepatic gene expression drug transporters and biotransformation enzyme expression and the other block contained hepatic transcription factor expression. Correlations between both blocks were performed using canonical correlations. Data is displayed as heat maps, with highest positive correlations considered as red and highest negative correlation, blue.

(Enriquez et al., 1999; Wang et al., 2003; Cheng et al., 2008). In males, Cyp2b10 mRNA levels increased with development of obesity compared with WT mice, whereas in female Cyp2b10 mRNA levels remained unchanged. These sex-specific changes in Cyp2b10 expression were observed with phenobarbital treatment, which is a known inducer of the Cyp2b family (Larsen et al., 1994; Cheng et al., 2008). Insulin treatment altered Cyp3a, 2e1, and 4a expression in hepatocytes (Kim et al., 2003), indicating that metabolism-related hormones can regulate phase-I enzyme expression. Our results indicate that in female mice, P450 enzyme mRNA expression during development of obesity correlates most closely with resistin and GLP-1, whereas in males these correlations are less well associated. Sexually dimorphic regulation of P450 enzyme expression might be responsible for sex-specific differences in expression and correlations with metabolism-related hormones (Hernandez et al., 2009).

Incretins, such as amylin and GLP-1, play a key role in insulin secretion and maintenance of blood glucose levels. In both male and female ob/ob mice, GLP-1 and amylin levels were increased compared with WT. Amylin is secreted along with insulin from pancreas and is involved in maintaining serum glucose levels (Moreno et al., 2011). Changes in amylin and insulin levels in our study were observed at the same time. Moreover, changes in amylin and insulin levels, along with resistin, showed significant correlation with other physiologic 
parameters analyzed in females during development of obesity. Hyperamylinemia was identified as one of the important factors causing several metabolic abnormalities during obesity and MetS (Reinehr et al., 2007). Drug therapies targeting incretins, such as GLP-1 agonists or DPPIV inhibitors, were developed as potential treatments for MetS (Schmitz et al., 2004). In humans, GLP-1 mimetic administration was shown to have drug-drug interaction by altering area under curve and $C_{\max }$ of drugs such as acetaminophen, lovastatin, digoxin, ethinyl estradiol, and lisinopril (Hurren and Pinelli, 2012). Although these changes were thought to be the result of delayed gastric emptying by GLP-1 mimetics, more detailed studies are needed to explain the identified changes in drug disposition. Correlation analyses in our study indicate that incretins such as GLP-1 and amylin may regulate DME and hepatic drug transporter expression, and changes in these incretin levels could potentially alter ADME of a drug via altered expression of DME and drug transporters.

Several studies have shown correlations between alteration in serum levels of various hormones during metabolic disorders. Studies also shown that hormones such as estrogen, progesterone, and androgens could alter hepatic uptake and efflux transporter expression (Geier et al., 2003; Kalabis et al., 2007). The role of metabolic hormone regulation of genes involved in drug metabolism is less well known. Acute or chronic administration of metabolism-related hormones, such as glucagon and GLP-2, can alter transporter expression and function. For example, GLP-2, an incretin secreted along with GLP-1, was shown to regulate Abcc2 expression in intestine (Villanueva et al., 2010). In human and rat hepatocytes, short term glucagon treatment altered Abcc3 function (Chandra et al., 2005). Our laboratory also showed glucagon treatment induces $\mathrm{ABCC} 2-4$ mRNA expression in human hepatocytes and that fasting can increase biliary excretion of Dibromsulphthalein (DBSP), an Abcc2 substrate (Kulkarni et al., 2014). Not only transporters but also phase-II DME expression were altered with glucagon and insulin treatment (Kim et al., 2003), indicating metabolism-related hormones have a potential to alter drug metabolism and related gene expression. Correlations observed in our study indicate that not only sex-related hormones, such as estrogen and testosterone (Lu et al., 1996; Geier et al., 2003), but also hormones involved in energy metabolism, such as resistin, glucagon, and GLP-1, are potential regulators of hepatic uptake transporter expression.

In summary, expression patterns in ob/ob mice were similar to WT littermates at week 1 . In both males and females, as obesity developed and metabolic hormone changes occurred, more robust expression changes in liver were observed after weeks 4 and 8. Drug transporter and DME expression were associated with metabolism-related hormones. Correlations revealed that the metabolism-related hormones, such as resistin, GLP-1, amylin, and glucagon were either coregulated or involved in regulation of hepatic gene expression, which needs further investigation. We recognize that species differences and use of mRNA data must be taken into consideration when extrapolating from our findings. This study was an initial step in exploring how clinical and serum measures can aid in the prediction of altered ADME processes and how metabolic hormone levels might regulate hepatic transporter expression during metabolic stress. Although this study did not identify a single biomarker for altered expression in hepatic drug transporters and DME expression during development of obesity, it did indicate that metabolic hormones may play role in regulating gene expression for processes that dictate drug metabolism and disposition. Identifying the roles of serum metabolic hormones, such as resistin, GLP-1, amylin, and glucagon, in regulating genes involved in drug metabolism is at present an unexplored field of study. Correlations observed in this study were obtained using mRNA levels, and future studies need to confirm these correlations at a protein and activity level. To summarize, this study provided insight into potential relationships between metabolic hormones and transporter expression, as well as insight into predicative markers for altered transporter expression.

\section{Authorship Contributions}

Participated in research design: Donepudi, Cheng, Slitt.

Conducted experiments: Donepudi, Cheng.

Contributed new reagents or analytic tools: Donepudi, Slitt.

Performed data analysis: Donepudi, Lu, Slitt.

Wrote or contributed to the writing of the manuscript: Donepudi, Lu, Cherrington, Slitt.

\section{References}

Aleksunes LM, Slitt AL, Maher JM, Dieter MZ, Knight TR, Goedken M, Cherrington NJ, Chan JY, Klaassen CD, and Manautou JE (2006) Nuclear factor-E2-related factor 2 expression in liver is critical for induction of $\mathrm{NAD}(\mathrm{P}) \mathrm{H}$ :quinone oxidoreductase 1 during cholestasis. Cell Stress Chaperones 11:356-363.

Aleksunes LM, Slitt AL, Maher JM, Augustine LM, Goedken MJ, Chan JY, Cherrington NJ, Klaassen CD, and Manautou JE (2008) Induction of Mrp3 and Mrp4 transporters during acetaminophen hepatotoxicity is dependent on Nrf2. Toxicol Appl Pharmacol 226:74-83.

Azuma K, Katsukawa F, Oguchi S, Murata M, Yamazaki H, Shimada A, and Saruta T (2003) Correlation between serum resistin level and adiposity in obese individuals. Obes Res 11: 997-1001.

Barshop NJ, Capparelli EV, Sirlin CB, Schwimmer JB, and Lavine JE (2011) Acetaminophen pharmacokinetics in children with nonalcoholic fatty liver disease. J Pediatr Gastroenterol Nutr 52:198-202.

Brill MJ, Diepstraten J, van Rongen A, van Kralingen S, van den Anker JN, and Knibbe CA (2012) Impact of obesity on drug metabolism and elimination in adults and children. Clin Pharmacokinet 51:277-304.

Chandra P, Zhang P, and Brouwer KL (2005) Short-term regulation of multidrug resistanceassociated protein 3 in rat and human hepatocytes. Am J Physiol Gastrointest Liver Physiol 288:G1252-G1258

Cheng Q, Aleksunes LM, Manautou JE, Cherrington NJ, Scheffer GL, Yamasaki H, and Slitt AL (2008) Drug-metabolizing enzyme and transporter expression in a mouse model of diabetes and obesity. Mol Pharm 5:77-91.

Cheng X, Maher J, Dieter MZ, and Klaassen CD (2005) Regulation of mouse organic aniontransporting polypeptides (Oatps) in liver by prototypical microsomal enzyme inducers that activate distinct transcription factor pathways. Drug Metab Dispos 33:1276-1282.

Cheng X, Buckley D, and Klaassen CD (2007) Regulation of hepatic bile acid transporters Ntcp and Bsep expression. Biochem Pharmacol 74:1665-1676.

Cocchi D, Parenti M, Cattaneo L, De Gennaro Colonna V, Zocchetti A, and Müller EE (1993) Growth hormone secretion is differently affected in genetically obese male and female rats. Neuroendocrinology 57:928-934.

Donepudi AC, Aleksunes LM, Driscoll MV, Seeram NP, and Slitt AL (2012) The traditional ayurvedic medicine, Eugenia jambolana (Jamun fruit), decreases liver inflammation, injury and fibrosis during cholestasis. Liver Int 32:560-573.

Dubuc PU (1976) The development of obesity, hyperinsulinemia, and hyperglycemia in ob/ob mice. Metabolism 25:1567-1574.

Enriquez A, Leclercq I, Farrell GC, and Robertson G (1999) Altered expression of hepatic CYP2E1 and CYP4A in obese, diabetic ob/ob mice, and fa/fa Zucker rats. Biochem Biophys Res Commun 255:300-306.

Faber KN, Müller M, and Jansen PL (2003) Drug transport proteins in the liver. Adv Drug Deliv Rev 55:107-124.

Geier A, Dietrich CG, Gerloff T, Haendly J, Kullak-Ublick GA, Stieger B, Meier PJ, Matern S, and Gartung C (2003) Regulation of basolateral organic anion transporters in ethinylestradiolinduced cholestasis in the rat. Biochim Biophys Acta 1609:87-94.

Grundy SM (2004) Obesity, metabolic syndrome, and cardiovascular disease. J Clin Endocrinol Metab 89:2595-2600.

Hardwick RN, Fisher CD, Street SM, Canet MJ, and Cherrington NJ (2012) Molecular mechanism of altered ezetimibe disposition in nonalcoholic steatohepatitis. Drug Metab Dispos 40:450-460.

Hernandez JP, Mota LC, Huang W, Moore DD, and Baldwin WS (2009) Sexually dimorphic regulation and induction of $\mathrm{P} 450$ s by the constitutive androstane receptor (CAR). Toxicology 256:53-64.

Hurren KM and Pinelli NR (2012) Drug-drug interactions with glucagon-like peptide-1 receptor agonists. Ann Pharmacother 46:710-717.

Kahn SE, Hull RL, and Utzschneider KM (2006) Mechanisms linking obesity to insulin resistance and type 2 diabetes. Nature 444:840-846.

Kalabis GM, Petropoulos S, Gibb W, and Matthews SG (2007) Breast cancer resistance protein (Bcrp1/Abcg2) in mouse placenta and yolk sac: ontogeny and its regulation by progesterone. Placenta 28:1073-1081.

Kim SK, Woodcroft KJ, and Novak RF (2003) Insulin and glucagon regulation of glutathione S-transferase expression in primary cultured rat hepatocytes. J Pharmacol Exp Ther 305: 353-361.

Kimura N, Hakamada K, Ikenaga SK, Umehara Y, Toyoki Y, and Sasaki M (2012) Gene expression of ATP-binding cassette transporters during liver regeneration after $90 \%$ hepatectomy in rats. Int J Mol Med 30:28-34.

Klaassen CD and Slitt AL (2005) Regulation of hepatic transporters by xenobiotic receptors. Curr Drug Metab 6:309-328.

Kulkarni SR, Donepudi AC, Xu J, Wei W, Cheng QC, Driscoll MV, Johnson DA, Johnson JA, Li $X$, and Slitt AL (2014) Fasting induces nuclear factor E2-related factor 2 and ATP-binding Cassette transporters via protein kinase A and Sirtuin-1 in mouse and human. Antioxid Redox Signal 20:15-30.

Laferrère B, Heshka S, Wang K, Khan Y, McGinty J, Teixeira J, Hart AB, and Olivan B (2007) Incretin levels and effect are markedly enhanced 1 month after Roux-en-Y gastric bypass surgery in obese patients with type 2 diabetes. Diabetes Care 30:1709-1716. 
Larsen MC, Brake PB, Parmar D, and Jefcoate CR (1994) The induction of five rat hepatic P450 cytochromes by phenobarbital and similarly acting compounds is regulated by a sexually dimorphic, dietary-dependent endocrine factor that is highly strain specific. Arch Biochem Biophys 315:24-34.

Lecureux L, Dieter MZ, Nelson DM, Watson L, Wong H, Gemzik B, Klaassen CD, and LehmanMcKeeman LD (2009) Hepatobiliary disposition of thyroid hormone in Mrp2-deficient TR rats: reduced biliary excretion of thyroxine glucuronide does not prevent xenobiotic-induced hypothyroidism. Toxicol Sci 108:482-491.

Lickteig AJ, Fisher CD, Augustine LM, Aleksunes LM, Besselsen DG, Slitt AL, Manautou JE, and Cherrington NJ (2007) Efflux transporter expression and acetaminophen metabolite excretion are altered in rodent models of nonalcoholic fatty liver disease. Drug Metab Dispos 35:1970-1978.

Lindström P (2007) The physiology of obese-hyperglycemic mice [ob/ob mice]. ob/ob mice ScientificWorldJournal 7:666-685.

Lu R, Kanai N, Bao Y, Wolkoff AW, and Schuster VL (1996) Regulation of renal oatp mRNA expression by testosterone. Am J Physiol 270:F332-F337.

Maher JM, Cheng X, Slitt AL, Dieter MZ, and Klaassen CD (2005) Induction of the multidrug resistance-associated protein family of transporters by chemical activators of receptor-mediated pathways in mouse liver. Drug Metab Dispos 33:956-962.

Mennone A, Soroka CJ, Cai SY, Harry K, Adachi M, Hagey L, Schuetz JD, and Boyer JL (2006) Mrp4-/- mice have an impaired cytoprotective response in obstructive cholestasis. Hepatology 43:1013-1021.

More VR and Slitt AL (2011) Alteration of hepatic but not renal transporter expression in dietinduced obese mice. Drug Metab Dispos 39:992-999.

Moreno P, Acitores A, Gutiérrez-Rojas I, Nuche-Berenguer B, El Assar M, Rodriguez-Mañas L, Gomis R, Valverde I, Visa M, and Malaisse WJ, et al. (2011) Amylin effect in extrapancreatic tissues participating in glucose homeostasis, in normal, insulin-resistant and type 2 diabetic state. Peptides 32:2077-2085.

Pantsulaia I, Livshits G, Trofimov S, and Kobyliansky E (2007) Genetic and environmenta determinants of circulating resistin level in a community-based sample. Eur J Endocrinol 156 $129-135$.
Pizarro M, Balasubramaniyan N, Solís N, Solar A, Duarte I, Miquel JF, Suchy FJ, Trauner M, Accatino L, and Ananthanarayanan M, et al. (2004) Bile secretory function in the obese Zucker rat: evidence of cholestasis and altered canalicular transport function. Gut 53:1837-1843.

Reinehr T, de Sousa G, Niklowitz P, and Roth CL (2007) Amylin and its relation to insulin and lipids in obese children before and after weight loss. Obesity (Silver Spring) 15:2006-2011.

Schmitz O, Brock B, and Rungby J (2004) Amylin agonists: a novel approach in the treatment of diabetes. Diabetes 53 (Suppl 3):S233-S238.

Slitt AL, Cherrington NJ, Maher JM, and Klaassen CD (2003) Induction of multidrug resistance protein 3 in rat liver is associated with altered vectorial excretion of acetaminophen metabolites. Drug Metab Dispos 31:1176-1186.

Starke AA, Erhardt G, Berger M, and Zimmermann H (1984) Elevated pancreatic glucagon in obesity. Diabetes 33:277-280.

Swerdloff RS, Batt RA, and Bray GA (1976) Reproductive hormonal function in the genetically obese (ob/ob) mouse. Endocrinology 98:1359-1364

Villanueva SS, Arias A, Ruiz ML, Rigalli JP, Pellegrino JM, Vore M, Catania VA, and Mottino AD (2010) Induction of intestinal multidrug resistance-associated protein 2 by glucagon-like Peptide 2 in the rat. J Pharmacol Exp Ther 335:332-341.

Wang Z, Hall SD, Maya JF, Li L, Asghar A, and Gorski JC (2003) Diabetes mellitus increases the in vivo activity of cytochrome P450 2E1 in humans. Br J Clin Pharmacol 55:77-85.

Wanless IR and Lentz JS (1990) Fatty liver hepatitis (steatohepatitis) and obesity: an autopsy study with analysis of risk factors. Hepatology 12:1106-1110.

Xu J, Kulkarni SR, Li L, and Slitt AL (2012) UDP-glucuronosyltransferase expression in mouse liver is increased in obesity- and fasting-induced steatosis. Drug Metab Dispos 40:259-266.

Address correspondence to: Dr. Angela L. Slitt, Department of Biomedical and Pharmaceutical Sciences, University of Rhode Island, 7 Greenhouse Road, Kingston, RI 02881. E-mail: aslitt@uri.edu 